\title{
COVID-19 Pathophysiology and Clinical Effects on Multiple Organ Systems - A Narrative Review
}

\author{
${ }^{1}$ Kinal Paresh Bhatt, MD, MPH., ${ }^{2}$ Jonathan Quinonez, DO., ${ }^{3}$ Abhinav Patel, MBBS., \\ ${ }^{4}$ Mehrie H. Patel, MBBS., ${ }^{5}$ Abdifitah Mohamed, MBBS., ${ }^{6}$ Muhammad Ali Aziz, MBBS., \\ ${ }^{7}$ Deepa Venkataramani, ${ }^{8}$ Fatema Zohora, MBBS., ${ }^{9}$ Namrata Hange, MBBS., MPH., ${ }^{10}$ Dana Awad, \\ ${ }^{11}$ Isha Mehta, MD., ${ }^{12} \mathrm{Paz}$ Ines M. Domingo, MD., ${ }^{13}$ Nadia Jamil., ${ }^{14}$ Shravika Reddy Kandi, \\ ${ }^{15}$ Sindhu Thevuthasan., ${ }^{16}$ Uzzam Ahmed Khawaja, ${ }^{1}$ Dhanya Baskaran, MD., \\ ${ }^{17}$ LaShathan Banks DO, MS, ${ }^{17}$ George Michel, MD., ${ }^{1}$ Dr Marcos Sanchez-Gonzales, MD., PhD. \\ ${ }^{1}$ Division of Clinical \& Translational Research, Larkin Health System, South Miami, FL, USA \\ ${ }^{2}$ The Haley Center, Winter Haven, FL, USA \\ ${ }^{3}$ Terna Medical College, Navi Mumbai, India \\ ${ }^{4}$ Pramukhswami Medical College, Gujarat, India \\ ${ }^{5}$ Shendi University, Shendi, Sudan \\ ${ }^{6}$ King Edward Medical University, Lahore, Pakistan \\ ${ }^{7}$ Aureus University School of Medicine, Oranjestad, Aruba \\ ${ }^{8} \mathrm{ZH}$ Sikder Medical College, Dhaka, Bangladesh \\ ${ }^{9}$ National University of Singapore, Singapore \\ ${ }^{10}$ Damascus University, Damascus, Syria \\ ${ }^{11}$ Windsor University School of Medicine, Cayon, St. Kitts \\ ${ }^{12}$ University of the East Ramon Magsaysay Memorial Medical Center, Quezon City, Philippines \\ ${ }^{13}$ Sargodha Medical College, Sargodha, Pakistan \\ ${ }^{14}$ Malla Reddy Institute of Medical Sciences, Hyderabad, India \\ ${ }^{15}$ Medical University of the America, Camps, St. Kitts \& Nevis \\ ${ }^{16}$ Jinnah Medical and Dental College, Abbottabad, Pakistan \\ ${ }^{17}$ Larkin Community Hospital, South Miami, FL, USA \\ *Corresponding author email: kinalbhatt@gmail.com \\ Received: 14 October 2020 / Revised: 04 May 2021 / Accepted: 20 May 2021 / Published: 12 June 2021
}

\begin{abstract}
Patients with comorbidities including Hypertension (HTN), Diabetes Mellitus (DM), Chronic Obstructive Pulmonary Disease (COPD), Asthma, Obesity, Cardiovascular Disease (CVD), Chronic Kidney Disease $(\mathrm{CKD})$, and those who are immunocompromised are prone to more severe complications of COVID-19 and a higher rate of hospitalizations. In the United States, around 94\% of COVID-19 deaths had an average of 2.6 additional conditions or causes per death. In a summary report published by the Chinese Centre for Disease Control and Prevention of 72,314 cases, casefatality rate was elevated among those with preexisting comorbid conditions- $10.5 \%$ for cardiovascular disease, $7.3 \%$ for diabetes, $6.3 \%$ for chronic respiratory disease, $6.0 \%$ for HTN, and 5.6\% for cancer. The COVID-19 pandemic continues to threaten people and healthcare systems globally and therefore the global economy. Currently, there is no cure or vaccine for COVID-19 and there is an urgent need to develop target therapies as we continue to learn more about this novel virus. Without therapeutic interventions, much of how we contain the viral spread is prevention through mitigation strategies (social distancing, face masks, supportive care). Early suspicion of COVID-19 symptoms with radiological and laboratory assessments may play a major role in preventing severity of the COVID19. With this literature review we aim to provide review of pathophysiology of Severe Acute Respiratory Syndrome Coronavirus-2 (SARS-CoV-2) and its clinical effects on multiple organ systems.
\end{abstract}

Keywords: COVID-19, pathophysiology, Clinical effects. 


\section{Introduction}

There are three major forms in which the Coronavirus disease 2019 (COVID-19) manifests, a gradual mild illness that is dominated by symptoms related to upper respiratory tract; non-life-threatening pneumonia; and a severe pneumonia with acute respiratory distress syndrome (ARDS) that starts with mild symptoms for around a week and subsequently deteriorates into ARDS, sepsis, and multiple organ failure $[1,2]$. According to the $\mathrm{CDC}$, the most frequent clinical manifestations include cough, dyspnea, fever, and sore throat. Patients have also reported new loss of taste and smell in other cases. COVID-19 affects almost every organ system in the body; due to its nature of transmission, it affects the lung tissue more so than others due to extensive distribution of angiotensin-converting enzyme-2 (ACE2) receptors. This can cause damage in the cardiovascular, renal, liver, central nervous system, gastrointestinal, and ocular systems that requires careful management $[3,4]$.

Patients with comorbidities such as HTN, DM, COPD, Asthma, Obesity, CVD, CKD, and malignancies are prone to more severe complications of COVID-19 and a higher rate of hospitalizations [5]. In the United States, around $94 \%$ of COVID-19 deaths had an average of 2.6 additional conditions or causes per death [6]. The current guidelines for minimizing the spread of COVID-19 involves the use of alcohol-based hand sanitizer [7, 8], thoroughly washing hands with soap and water, maintaining $1 \mathrm{~m}-2 \mathrm{~m}$ (3-6ft) with others, self-isolation of people who were exposed, or are experiencing symptoms, and practicing good respiratory hygiene [7]. Public masking has been widely accepted as one of the most important pillars of controlling the COVID-19 pandemic $[9,10]$.

\section{Epidemiology}

The first case of COVID-19 was reported in Wuhan, China in early December 2019 [11]. The number of COVID-19 cases reported to the WHO has been increasing since then. A month later, on January 20th, 2020, WHO reported that there was a total of 282 confirmed cases of
COVID-19 in four countries including China (278 cases), Thailand (2 cases), Japan (1 case) and the Republic of Korea (1 case) [12]. During the initial stages of COVID-19 spread, most cases reported globally that were outside of China, and therefore attributed to travelers who were infected in China and then travelled outside the country [13]. The first case of human-to-human transmission of COVID-19 in the US was reported in Washington State on January 20th, 2020 in a patient who had returned from Wuhan, China after visiting his family [14]. The virus then rapidly spread throughout the country and the United States quickly became an epicenter with New York reporting the most cases $(475,000$ cases reported as of October 9, 2020) [15]. The first death in the US due to COVID19 was reported on April 13th, 2020 in Wisconsin, with New York reporting most deaths 32,859 death reported as of October 9, 2020) at later stages of the pandemic [15].

According to the WHO, by March 2020, during the initial stages of the pandemic, SARS-CoV-2 had spread to over 100 countries, with over 100,000 infections and over 3,500 deaths and an estimated case fatality rate was $0.9 \%-3 \%$ [16]. As the pandemic progressed, according to the data reported by WHO on September $13^{\text {th }}$, there are a total of 28,637,952 confirmed cases globally, with a total of 917,417 number of deaths across 216 countries [17]. With $51 \%$ of the total cases reported in the Americas, it has the highest number of cases among the WHO regions. At the beginning of the outbreak the infection followed exponential growth where the infection cases doubled in size every 7.4 days. The basic reproduction number was from 2.24 to 3.58, which is higher than SARS [18]. All age groups are likely to be affected by COVID-19 with majority of patients between 36 to 65 years of age [19].

\section{Mechanism of SARS-CoV2}

Zhang et al. (2020) demonstrated that all coronaviruses $(\mathrm{CoV})$ have an analogous spike structure on their surfaces. In SARS-CoV-2, part of the spike (S) protein on the virus is used to bind to a receptor on the host cell and to fuse through the cell membrane. The receptor for 
Bhatt et al., Int. Ann. Sci.; Vol. 10, Issue 1, pp: 151-167, 2021

Severe Acute Respiratory Syndrome Coronavirus-2 (SARS-CoV-2) is the angiotensinconverting enzyme 2 (ACE2) [20]. After binding to ACE2, the $\mathrm{S}$ protein is cleaved and prepared for the next steps - fusion and entering the cell where endocytosis plays a major role in completing the subsequent steps [20]. Following that, the viral genome is exposed and translated inside the host's cell, all of the translated proteins that form the virus' particles aggregate inside the endoplasmic reticulum-Golgi intermediate compartment, and then they are constituted in specific small vesicles in order to be transported to the extracellular compartment, thus infecting other cells [20]. In terms of receptor affinity, many studies have pointed out that the affinity of SARS-CoV-2 to human ACE2 is higher than that of Severe Acute Respiratory Syndrome Coronavirus (SARS-CoV), which makes SARSCoV-2 more contagious between people [20].

While angiotensin-converting enzyme (ACE) yields angiotensin II (Ang II) from angiotensin 1 (Ang 1), 106 ACE2 yields Ang 1-7 from Ang II; Ang II causes vessels to constrict and induces proliferation 107 (Angiogenesis), while Ang 1-7 renders vessel dilation and limits cell growth/replication. When the balance 108 between Ang II and Ang 1-7 is disturbed (Ang II outweighs Ang 1-7), acute lung injury will occur, and this is 109 what happens when SARS-CoV-2 binds to ACE2, a receptor found on nearly all cells in the human body, 110 decreasing the production of ACE2 in the cell [20]. A genetic study demonstrated that ACE2 contains many 111 genes that enhance viral strength during its lifecycle, and it concluded that ACE2 production increases SARS112 CoV-2 viral load in the organs of the body [20].

\section{Clinical Effects of COVID-19}

\subsection{Clinical effect of SARS-CoV-2 on Respiratory system}

Hu et al. (2020) conducted a metanalysis of 21 published trials to evaluate prevalence of COVID-19 symptoms, comorbidity, complications, and other outcomes in patients in China and Singapore [21]. The meta-analysis revealed hypertension and diabetes were the most common comorbidities associated with increasing severity of the SARS-CoV-2. However, the main obstacles in the treatment recovery were found to be ARDS and acute lung injury (ALI). As of June 25, 2020, the CDC also provided list of health conditions that are detrimental to severity of COVID-19 illness: moderate to severe asthma, pulmonary fibrosis, smoking and immunocompromised state due to corticosteroid use or immunosuppressant medications [21]. An Ex-vivo study conducted by Chu et al. (2020) compared infection and replication of SARS-CoV-2 and SARS-CoV in human lung tissues. The study revealed SARSCoV-2 is 3.20-fold more infectious than SARS$\mathrm{CoV}$ in human lung tissue [22].

Primary cause of death in COVID-19 is progressive respiratory failure secondary to diffuse alveolar damage [23]. Human lung cells normally express ACE2 receptors. Ackermann et al. (2020) examined 7 autopsied lungs from known positive COVID-19 patients and compared them with uninfected patients who died from ARDS related to influenza A (H1N1) and uninfected control lungs [23]. The study revealed immunohistochemical analysis of ACE2 in COVID-19 infected group, measured in Standard Deviation $( \pm S D)$ as "cells per field of view" were $0.25 \pm 0.14$ in comparison to $0.35 \pm$ 0.15 and $0.053 \pm 0.03$ in influenza and uninfected control group, respectively. Furthermore, ACE2 positive lymphocytes were not discovered in the alveoli of the control group but were present in both COVID-19 and Influenza groups, slightly higher in COVID-19 group [23]. Research has now shown ACE2 involvement in COVID19 is not limited to a role of a receptor on host cell where COVID-19 binds and enters the cell, but also involved in regulation of the infection, including immune response with cytokine secretion and replication of viral genome [24].

ARDS is caused by direct or indirect injury to the lung because of an acute systemic inflammatory response triggered by COVID-19 [24]. Studies reported dry cough (59.4-82\%) as the most common respiratory manifestation of COVID19, which suggests damage to epithelial lining as there is no sputum production [25]. Although COVID-19 affects multiple organ systems, the 
incidence of ARDS (15.6-31\%) is higher in comparison to other organ injuries. This is secondary to damage to epithelial cell lining of the alveolar cells. Endothelial cells are the deeper layer of blood vessels in all organs, and therefore it is possible that these cells are not involved in earlier manifestation of COVID-19, therefore other organs are less involved [25]. Patients with low oxygen index are considered to have severe respiratory failure and will need chest $\mathrm{CT}$ imaging (Xray and CT), which may show a mixed pattern of ground-glass appearance and consolidation [25]. However, despite severity seen on imaging, patients may not be experiencing dyspnea and have stable tissue perfusion, indicated by normal lactate level. Despite inconsistency between laboratory, imaging and symptoms experienced, these patients are to be monitored closely, as their condition may deteriorate rapidly [25]. Blood carbon dioxide is used as an indicator for invasive mechanical ventilation $[25,26]$.

\subsection{Clinical effect of SARS-CoV-2 on Cardiovascular system}

SARS-CoV-2, which causes COVID-19, is a novel virus of interest for the cardiovascular system as it causes deleterious effects to a host of higher-risk populations. Persons in higher-risk populations include those who have coronary artery disease, diabetes mellitus, hyperlipidemia, hypertension, and obesity [27, 28, 29]. Age is another risk factor of consideration as an elderly individual ( $x>60$-years-old) infected with SARS$\mathrm{CoV}-2$ developed more systemic complications [29]. Any individual with the above risk factors and infected with this virus will have a poor prognosis due to increased expression of angiotensin-converting enzyme 2 and an increased propensity for cytokine storm [27, 29]. The mechanism of action of cardiovascular damage once SARS-CoV-2 binds to ACE2 is as follows: ACE2 is normally expressed in the heart, kidneys, lungs (particularly type-2 alveolar cells), and other organs $[27,28,29]$. Patients with the above risk factors and on appropriate antihypertensive therapies (lisinopril, losartan) affecting the renin-angiotensin-aldosterone system (RAAS) will have increased expression of ACE2. Once SARS-CoV-2 binds to ACE2, this action alters ACE2 signaling pathways leading to vasodilatation and suppressed cell growth. Acute lung injury, in the form of severe pneumonia, puts stress on the heart leading to acute hypoxia and decompensation [28, 29]. As a result, coronary arterial flow increases to keep up with increased myocardial oxygen demand. Lack of oxygen from decreased blood flow leads to direct myocardial damage (apoptosis), whereas increased shear stress upon coronary arteries can result in plaque rupture and eventually myocardial infarction $[28,29$. At the same time this occurs, the immune system responds with systemic inflammation through the release of pro-inflammatory cytokines (Interleukins 1 and 6, tumor necrosis factor-alpha) resulting in systemic inflammation, multiorgan failure, and depressed myocardial function [29]. Other important causes of decreased myocardial function include the development of acute coronary syndrome, acute myocarditis, disseminated intravascular coagulation (procoagulation state), electrolyte imbalances (hypokalemia), shock (cardiogenic), and stressedinduced cardiomyopathy [29].

A workup for COVID-19 will reveal that patients will either be asymptomatic or present with a variety of symptoms [30]. Common symptoms of COVID-19 include anorexia, a dry cough, dyspnea, fever, fatigue, and myalgia [30]. Concerning the cardiovascular system, a patient may present with chest pain, dyspnea, and heart palpitations. A workup for acute coronary syndrome begins once a patient presents for further evaluation $[28,29]$. Workup will reveal an elevated cardiac troponin I (cTnI) and an abnormal EKG demonstrating either ST elevation or depression, along with brady/tachyarrhythmias [29]. In the setting of acute hypoxia, lab work may show an elevated Ddimer, fibrin degradation products, and fibrinogen [27]. Severely elevated values of cTnI or $\mathrm{D}$-dimer indicate a poor prognosis. Once a patient has severe COVID-19 as a confirmed diagnosis and is transferred to the intensive care unit (ICU) setting, the disease course usually worsens. ICU patient may exhibit acute heart failure, elevated blood pressure, and persistent c'TnI elevation. When a patient becomes 
discharged from the hospital setting, patients may experience abnormalities with glucose and lipid metabolism [27].

Management will depend on disease severity. Hospital admissions will require the following considerations [27, 28, 29]:

- Anticoagulation: Heparin or lowmolecular-weight heparin for seven days. Drug-drug interactions should be monitored with concomitant use of antiviral drugs. Low-molecular-weight heparin is preferred in the ICU setting.

- Anti-hypertensives: RAAS inhibitors (lisinopril, losartan) should be continued in the hospital setting if previously prescribed. There is some controversy regarding higher ACE2 expression.

- Immune modulating drugs: Prednisone and IL-6 inhibitors (Tocilizumab) should be considered only when the case is severe and cytokine storm is imminently anticipated.

- Mechanical cardiopulmonary support: Further research is required given variable survival rates with modalities, not limited to extracorporeal membrane oxygenation (ECMO), intra-aortic balloon pump, and veno-arterial ECMO.

- Testing: Testing should only be warranted if it contributes to meaningful management of complications of COVID-19.

Follow-up in the outpatient setting will need constant monitoring of blood pressure, glucose, and lipid regulation [27].

\subsection{Clinical effect of SARS-CoV-2 on Immune System}

Cytokine storm is an informal term that refers to the dysregulated release of proinflammatory cytokines as a reaction to infection and various stimuli [31]. In a wider sense, cytokine storm may pertain to hyperactivity in the immune response as it entails releasing various mediators such as interleukins, interferons, chemokines, tumornecrosis factors, and other mediators [32]. During the ongoing COVID-19 pandemic, there has been an increasing number of severe COVID-19 cases deteriorating as a result of a cytokine storm. Critically ill or severely ill patients deteriorate suddenly in the later stages of COVID-19 or the recovery phase. An important challenge is to distinguish a dysregulated inflammatory response from an appropriate inflammatory response in the pathophysiology of COVID-19. This is due to the implications surrounding how "cytokine storm" is defined. When discussing the cytokine storm in the context of an inflammatory response, it is implied that increasing cytokine release is damaging to host cells [32]. Patients who are at risk for developing cytokine storm include those with comorbid illnesses including coronary artery disease, obesity, cancer, primary immunoglobulin deficiencies, autoimmune conditions, and recipients of immunosuppressive therapies [33]. Patients with these underlying comorbid conditions are at higher risk of developing severe COVID-19 and concurrent with this, an increased risk of developing a cytokine storm. As such, special consideration must be given to these high-risk populations when it comes to guidelines in their management when infected with COVID-19.

SARS-CoV-2 is a novel virus of interest for the immune system as it causes immune dysregulation. Once infected with SARS-CoV-2, it manifests a myriad of symptoms in a patient. $\mathrm{A}$ patient can be asymptomatic or exhibit anorexia, a dry cough, dyspnea, fever, fatigue, and myalgia. Such symptoms are associated with a high rate of human-to-human transmission. Several cytokines implicated in the production of these symptoms include interleukins 2 (IL-2) and 6 (IL-6) as well as tumor necrosis factor-alpha (TNF-a). A consequence of persistent IL-2 levels is capillary leak syndrome which is characterized by increasing capillary permeability to proteins leading to edema, hypotension, acute respiratory failure, and kidney injury [34].

Relevant evidence from severe cases of COVID19 suggests that proinflammatory responses play a role in the pathogenesis of this disease [34]. Several proinflammatory cytokines (IL-6, IL-8, IL-1 $\beta, \quad$ granulocyte-macrophage colonystimulating factor, and reactive oxygen species), 
and chemokines (CCL2, CCL-5, IFN $\gamma$-induced protein 10, and CCL3) contribute to the occurrence of Acute Respiratory Distress Syndrome (ARDS). These proinflammatory responses contribute to cytokine and chemokine response dysregulation in the setting of high virus titers following SARS-CoV-2 infection, leading to an inflammatory cytokine storm. This inflammatory cytokine storm is most often accompanied by immunopathological changes in the lungs [34]. Immunopathological lung changes may be a result of rapidly increasing cytokines and chemokines attracting numerous inflammatory cells such as neutrophils and monocytes. These neutrophils and monocytes, in turn, cause an excess in inflammatory cell infiltration into lung tissue, resulting in lung injury [34]. It can be postulated from these immunopathological lung changes that dysregulated cytokine and chemokine responses by SARS-CoV-2 infected cells play an essential role in the pathogenesis of COVID-19.

A distinguishing factor in cytokine storm is persistent fever and constitutional symptoms such as joint pain, muscle pain, weight loss, headache, and fatigue. When a cytokine storm occurs, systemic inflammation spreads across different organ systems leading to manifestations such as loss of vascular tone presenting as hypotension, vasodilatory shock, and progressive organ failure [34]. ARDS results and contributes further to multiple-organ failure in quick succession, resulting in sudden death. Cytokine storm suppression is of utmost importance to prevent detrimental deterioration of COVID-19 patients thus increasing their chances of recovery [31]. Sun, X. et al. (2020) reported a retrospective study of 41 COVID-19 patients which showed patients presented with a myriad of symptoms ranging from an asymptomatic state to ARDS [35]. Severe complications associated with COVID-19 include ARDS, a "Kawasaki-like syndrome" in children, inflammatory myocarditis, myocardial infarction, cardiac arrest, multiple organ failure, ischemic stroke, secondary hemophagocytic lymphohistiocytosis, acute pulmonary embolism, bacterial pneumonia [35].

\subsection{Clinical effect of SARS-CoV-2 on Renal system}

Most patients with COVID-19 have mild symptoms, but about 5\% develop severe symptoms, which can include acute respiratory distress syndrome, septic shock, and multiple organ failure. Patients with kidney disease exhibited worse clinical outcomes when infected with SARS-CoV-2 [36]. In addition to COVID19 causing AKI in $5-15 \%$ of the cases, it also impacts patients with chronic kidney disease (CKD), patients on chronic replacement therapies, and patients with a kidney transplant. Patients with end-stage renal disease (ESRD) are particularly vulnerable to severe COVID-19 due to the older age and high frequency of comorbidity, such as diabetes and hypertension, in this population [37].

The ACE2 receptor is normally expressed in the heart, kidneys, lungs (particularly type- 2 alveolar cells), and other organs [27]. The SARS-CoV-2 binds to this receptor causing direct cellular injury resulting from viral entry through the ACE2 receptor, which is highly expressed in the kidney. The virus's deleterious effects on the kidney are thought to be multi factorial including a wide range of potential mechanisms. Targeting of ACE2 by SARS-CoV-2 combined with its selective tropism for kidneys, results in angiotensin dysregulation, innate and adaptive immune pathway activation, and hypercoagulation to result in tubular injury and AKI associated with COVID-19.

Organ crosstalk between the injured lungs, the heart, and the kidney may further propagate injury [38]. There seems to be a close relationship between alveolar and tubular damage due to cytokine overproduction, which establishes the "lung-kidney axis" in ARDS. ARDS also may cause renal medullary hypoxia, which is an additional insult to tubular cells [39]. Cardiomyopathy and acute viral myocarditis seen in severe COVID-19 cases can both contribute to renal venous congestion, hypotension and renal hypoperfusion, leading to a reduction in glomerular filtration rate (GFR) [40]. The resulting fluid imbalances contribute to pre-renal AKI. The pro-inflammatory cytokines elicited by 
the viral infection or the resultant rhabdomyolysis cause toxic tubular damage predominantly affecting the proximal convoluted tubules (PCT). Non-specific mechanisms include drug nephrotoxicity, high levels of PEEP in patients requiring mechanical ventilation, and nosocomial sepsis. The interactions may facilitate Acute kidney injury (AKI) commonly seen in critically ill patients, affecting $\sim 20-40 \%$ of patients admitted to intensive care, and is considered as a marker of disease severity and a negative prognostic factor for survival [41]. Several studies have highlighted changes in urinary sediment, including proteinuria and hematuria, and evidence of urinary SARS-CoV-2 excretion, suggesting the presence of a renal reservoir for the virus [42].

Although vaccine has been developed against the SARS-CoV-2 virus, the critical aspect of management is supportive care. Implementation of the Kidney Disease: Improving Global Outcomes (KDIGO) supportive care guideline (e.g., avoidance of nephrotoxins, regular monitoring of serum creatinine and urine output, consideration of hemodynamic monitoring) in critically ill patients with kidney involvement is likely to reduce the occurrence and severity of AKI in COVID-19. The evaluation of AKI in COVID-19 patients should follow the general approach to workup and diagnosis of AKI, utilizing the broad framework of prerenal, intrarenal, and postrenal causes.

In patients with suspected or confirmed COVID19 who develop AKI, an emphasis should be placed on optimization of volume status to exclude and treat prerenal (functional) AKI, while avoiding hypervolemia, which may worsen the patient's respiratory status. If conservative management fails (volume management, discontinuation of nephrotoxic medications, etc.), renal replacement therapy (RRT) should be considered in patients with volume overload, especially those with refractory hypoxemia. In patients with COVID-19 and AKI, early initiation of RRT and sequential extracorporeal organ support (ECOS) seem to provide adequate organ support and prevent progression of disease severity [43]. In the patients who developed AKI from COVID-19 infection, around $1.5-9 \%$ of them required RRT in the form of continuous renal replacement therapy (CRRT) [44].

\subsection{Clinical effect of SARS-CoV-2 on Diabetes}

One of the organs affected in COVID19 is the pancreas. As it has come to be a known observation that patients who are suffering from Diabetes Mellitus (DM) tend to have more severe outcomes due to COVID-19 disease in addition to being more susceptible to acquiring COVID19 infection [45]. Due to these reasons, Lian et al. conducted a study in which they divided the study population into two groups: younger than 60 years and older than 60 years. The authors showed that a variety of comorbidities, including $\mathrm{DM}$, were more commonly found in the older than 60 years group [46]. Therefore, the age difference is most likely one of the most important reasons for risk that is attributed to diabetic patients with COVID-19 [45]

A study by Hoffman et al. (2020) suggested that ACE2, converts angiotensin II to angiotensin I-7 which also causes vasodilation, is the receptor used by the Coronavirus (SARS-CoV-2) to bind to their target cells which is expressed by the epithelial cells of the lungs, intestine, kidney and blood vessels [47]. As mentioned in Fang et al. (2020), patients with types I and II DM who are treated with ACE inhibitors and Angiotensin II type-I receptor blockers (ARBs) demonstrated increased expression of ACE2. There has been a noted increase in ACE2 expression by Thiozolidinadiones. Given that DM is a hyperinflammatory condition, Tadic et al. (2020), mentions a few possible mechanisms for DM being an independent risk factor for COVID-19 infection. First being inflammation, secondly, hypercoagulable state, and last but not the least, dysfunctional sympathetic nervous system (SNS) and activated Renin-Angiotensin-Aldosterone system (RAAS) [45]. It is known that RAAS function is already significantly modified in diabetics [47]. Therefore, in summation, a diabetic person would have more expression of ACE2 as such, on the treatment of ACEI and/or $\mathrm{ARBs}$, in conjunction with certain anti-diabetic medications that can demonstrate increased susceptibility to SARS-CoV-2 which acts through 
ACE2. Therefore, when a diabetic, with altered RAAS, is infected with COVID-19, he or she may undergo "acute change in the activity of ACE2" which can cause a trigger for the hypercoagulable and hyper-inflammatory state [48]. Guo et al. (2020)'s case-control study suggested that patients with DM in absence of other comorbidities infected by SARS-CoV-2 still demonstrated a "higher risk of severe pneumonia, uncontrolled inflammatory response, higher levels of tissue injury-related enzymes, and higher hypercoagulable state" [49, 50]. They also found that the serum inflammatory biomarkers levels were significantly raised in diabetics in comparison to those without DM; these biomarkers include C-reactive protein, Ddimer, IL-6, serum ferritin, and coagulation index [50].

Muniyappa et al (2020) also discusses potential mechanisms that may play a role in increasing the susceptibility for COVID-19 in patients with DM. Munniyapa et al. mentions 5 main mechanisms; "1) higher affinity cellular binding and efficient virus entry, 2) decreased viral clearance, 3) diminished $\mathrm{T}$ cell function, 4) increased susceptibility to hyperinflammation and cytokine storm syndrome, and 5) the presence of CVD" [51]. In addition to ACE2, SARS-CoV-2 also acts through another receptor DPP4. Now it has come to understanding in two meta-analyses studies that DPP4 inhibitors, which are widely used in diabetics, increase the risk for a variety of infections, whereas " a third meta-analysis showed that there is no increased risk of infections with DPP4 inhibitors". For these reasons, it is highly important to study whether the DPP4 inhibitors would increase the susceptibility or severity of SARS-CoV-2 infection by conducting clinical trials [52].

In the current COVID-19 pandemic, due to lockdowns, diabetic management is now difficult due to travel restrictions, however, Singh $A K$ et al. mentions modern innovations like telemedicine that can be specifically crucial for diabetes management [53]. It is important to note that glycemic control is very important in diabetic patients with COVID-19. Professional societies such as the American Association of Clinical Endocrinologists and the European society of endocrinology have been in agreement regarding the need for people with Diabetes to prevent and prepare for the spread of COVID-19 by taking regular precautions such as staying home as much as possible and washing hands regularly as well as practising social distancing. In the gist of the current COVID-19 pandemic, current guidelines advise people with Diabetes Mellitus to continue taking their medication in order to maintain glucose control. Moreover, the guidelines also suggest stocking up on an additional 30-day supply of medication and supplies to be able to monitor blood glucose levels at home $[55,55,56]$. The International Diabetes Federation has mentioned that people with diabetes are amongst the high-risk categories that can have a serious illness if they contract the SARS-CoV-2, however, it is best not to rush to the hospital in order to contain the virus [53]. National Health Service (NHS) clinical guidelines for the management of diabetic patients in COVID-19 recommend expedition of treatment and discharge of inpatients and implementation of the virtual clinics/teleconsultations [54, 55, 56]. However, NHS fails to address the challenges which might be faced in regards to literacy, poor access and for people who might be not wellversed with technology. Another useful consideration could be to discontinue SGLT-2 inhibitors due to the risk of dehydration and euglycemic ketosis [53]. Singh AK et al, further suggest that metformin may also need to be stopped if a diabetic patient experiences vomiting or poor oral intake [53]. Other anti-diabetic medications that include sulfonylureas and insulin may have to be altered depending upon blood glucose levels [53]. Diabetic patients should maintain a regular diet consisting of balanced amounts of protein, fiber, and limited saturated fats, which are crucial to maintaining good glycemic control. Regular indoor exercise is also beneficial to good glycemic control [52]. Medication use should also be adhered as well [53]. Telemedicine is highly valuable for diabetic patients and they should be explained that telemedicine services are available to them to consult their physician regularly. Diabetic patients must be educated on foot care as well to avoid diabetic-related complications [53]. In 
summary, diabetic patients must receive proper education on proper glycemic control and strict medication use to maintain their DM [53].

Around the globe, there are many religions and communities that practice fasting. It has been a known fact that fasting has been a concern in Diabetics as fasting can cause hypo- or hyperglycemia in Diabetics. This is particularly of major concern given the ongoing COVID-19 pandemic. Therefore, Ahmed, W. N. et al. (2020), mentions in their review that people with diabetes should go through systemic and structure-based management during fasting amidst the pandemic [54]. It is recommended that Family Physicians conduct a risk stratification for their diabetic patients who are wanting to fast during the pandemic; then to recommend an individual whether fasting would be advisable for them or not [54]. All other measures are the same as for non-fasting diabetics, ie., to have regular teleconsultations, proper dose modifications, and regular glucose home monitoring [54]. In summary, diabetic patients must receive proper education on proper glycemic control and strict medication use to maintain their DM.

\subsection{Clinical effect of SARS-CoV-2 on Gastrointestinal system}

As per studies by Gu et al. (2020), Cheong et al. (2020), Patel et al. (2020), nearly 50\% of COVID19 patients have presented with concurrent gastrointestinal (GI) manifestations like diarrhea (most common), nausea, vomiting, and abdominal pain; in some cases, anorexia, dysgeusia, anosmia, and hematochezia have also been reported [50, 57, 58]. The demographics that are most vulnerable to pulmonary and extrapulmonary (like gastrointestinal) manifestations include older adults, smokers, HIV patients, immunocompromised, and those who suffer from comorbidities [59]. Moreover, patients with underlying inflammatory bowel disease (IBD)are also at increased risk of contracting COVID-19 [50, 60]. The GI manifestations in COVID-19 are noted to be present in both adult and pediatric populations ranging from 10 months to 78 years old, with an average age of 46.1 years [50]. It is also important to note, there was a higher incidence of vomiting seen in children as compared to adults even though diarrhea was the most common symptom in both these populations [50].

Based on recent studies of GI manifestations in COVID-19 patients, it has been commonly hypothesized that the infection can be transmitted through the fecal-oral route. Besides confirming the presence of SARS-CoV-2 RNA in feces, also confirmed the long periods of viral shedding lasting approximately two weeks to over a month, supporting the hypothesis of viral replication occurring in the GI tract [50]. Additionally, the presence of the SARS-CoV-2 RNA in stool was noted to be independent of its presence in the respiratory samples since it was found that the virus can continue to be present in the fecal matter even after the respiratory symptoms have subsided and the respiratory specimens (including nasopharyngeal swabs) have tested negative for SARS-CoV-2 [50]. SARS-CoV-2 is likely to be transmitted by both symptomatic as well as asymptomatic patients causing GI infectivity with the incubation period estimated between 2 to 14 days [59].

Cha et al. (2020) concluded an association between pathogen, host, and environmental factors that can enhance the SARS-CoV-2 infectivity through the fecal-oral transmissibility. Although environmental influences such as close contact with infected persons (including symptomatic and asymptomatic patients), poor personal hygiene, and poor public sanitation play a significant role in infectivity, GI symptoms and their corresponding severity depend on the pathogen load in stool and saliva, viral shedding, the viability in the environment, infectious dose [59], and the extent of viral gene expression in enterocytes and stratified epithelial cells of the esophagus, which can cause the direct intestinal injury and infectivity [60]. SARS-CoV-2 can lead to GI manifestations through its interaction with specific cell receptors called ACE2, and host cellular transmembrane serine protease(TMPRSS) which, besides in the lungs pneumocytes, are also expressed in stratified epithelial cells of the esophagus, as well as absorptive enterocytes in the ileum and colon of the intestine [57, 60,61]. This inflammatory response caused by SARS-CoV-2 infection leads 
to direct injury to the enteric system including its absorptive and epithelial enterocytes; consequently, resulting in malabsorption, and secretory diarrhea [50].

The study conducted by Baryah et al. (2020) and Patel et al. (2020) has suggested for fecal and saliva specimen testing in COVID-19 patients who have reported GI symptoms by confirming identification of the viral nucleic acid (SARSCoV-2 RNA) in the stool and salivary samples through real-time reverse transcriptasepolymerase chain reaction (rRT-PCR technique) $[50,60]$. In addition, they briefly mentioned that endoscopy and biopsy had further confirmed the presence of SARS-CoV-2 nucleocapsid protein in the enteric epithelium including, the esophagus, stomach, duodenum, and rectum. However, such diagnostic tools including, peg tube procedures, endoscopy, and colonoscopy can potentially pose a threat to viral transmission, thus limiting their usage $[50,59]$.

It is crucial to rule out other infectious etiologies including Clostridium difficile before treating COVID-19 patients for their enteric symptoms. Nausea and vomiting are managed conservatively with antiemetic medications, and in cases of bacterial coinfection, antibiotics are administered. Management is mainly focused on effective infection control through the use of Personal Protective Equipment (PPE), keeping the close contacts informed about the viral shedding (even during incubation and asymptomatic phase), encouragement of proper hygiene and hand-washing practices, and most importantly the adherence to social distancing guidelines [50].

Globally, a total of 326 patients with underlying inflammatory bowel disease (IBD) have reported being infected with COVID-19, 189 of which had Crohn's disease, while 135 cases had ulcerative colitis [59]. A study by Baryah et al. (2020) documented the upregulation of receptors like ACE2 and TMPRSS, and increased cytokine storm in patients with IBD, making them vulnerable to contracting COVID-19, and an increased likelihood to report increased severity in symptom manifestation [60]. Thus, precautionary measures recommended to patients with IBD include proper hand hygiene,
PPE, face masks, avoidance of hospital settings, and most importantly to adhere to social distancing guidelines in public settings. Cha et al. (2020) and Baryah et al. (2020) in their recent studies summarized that IBD patients who were managed by immunomodulatory agents that attenuate the inflammatory response may protect them from contracting COVID-19 as well as developing severe conditions; however, since they act as immunosuppressants, their recommendations may vary during an active SARS-CoV-2 infection depending on the medications, presence, and severity of SARSCoV-2, as well as IBD flare-up [59, 60]. In these cases, patients may need to continue with their ongoing regiments of 5 aminosalicylic acid (5ASA) or may be asked to withhold some treatments (including prednisone if dose is $\geq 20$ $\mathrm{g} / \mathrm{d}$, tumor necrosis factor, and other immunomodulators and immunosuppressants) if positive for COVID-19, and reinstated after the viral clearance [59, 60, 61].

Most of the studies discussed were conducted in China, thus the applications of these findings and recommendations on a global level are limited. There is also a variance between the findings of the studies discussed. Although the studies hav confirmed the presence of SARS-CoV-2 in the fecal matter and subsequent viral shedding, there is no proven evidence to confirm the fecal-oral transmission and replication of the virus. More studies need to be conducted; however, current findings have provided promising insights into the critical effects of COVID-19 on the GI system.

\subsection{Clinical effect of SARS-CoV-2 on Liver}

Liver injury associated with COVID-19 is defined as any clinical or laboratory abnormality related to liver function that appears in patients either with or without a pre-existing liver disease [62]. Liver function derangements with Covid-19 infection are usually transient and mild. The most frequent derangements observed is an elevated Alanine aminotransferase (ALT) and Aspartate transferase (AST) level, with the incidence ranging from $2.5 \%-50.0 \%$ and $2.5 \%-61.1 \%$ respectively. Total bilirubin levels are elevated 
Bhatt et al., Int. Ann. Sci.; Vol. 10, Issue 1, pp: 151-167, 2021

from $0 \%-35.3 \%$ of Covid-19 positive patients. Elevated alkaline phosphatase (AKP) and gamma-glutamyl transferase (GGT) levels have been reported in patients with comorbid NAFLD and this was associated with a more severe disease course and a poor prognosis [4758].

Studies have found moderate microvascular steatosis, mild lobular and portal activity, mild sinusoidal dilatation and an increase in mitotic cells and ballooned hepatocytes on liver biopsy of Covid-19 patients [69, 70, 71]. A few mechanisms have been proposed to explain the liver injury patterns.

1. Cytotoxicity: The SARS-CoV-2 may directly infect hepatocytes and cholangiocytes - since the receptor of the virus, ACE2 is present on both types of cells and induce apoptosis via a caspasedependent pathway [62]. It is important to note, however, that biopsy of liver tissue from a patient who died from COVID-19 has shown no viral inclusions [62-69].

2. Cytokine storm: Several studies have reported that COVID-19 patients with abnormal liver function have higher serum pro-inflammatory cytokine and chemokine levels including $\mathrm{C}$ reactive protein (CRP), procalcitonin, serum ferritin, LDH, D-dimer, IL-6, IL-2 and lymphopenia - a consequence of immune activation [62-69]. This suggests that the liver injury seen in SARS-CoV-2 might be a consequence of the cytokine storm.

3. Hepatotoxic drugs: the use of hepatotoxic drugs including lopinavir, ritonavir, remdesivir, chloroquine, tocilizumab, uminefovir, Chinese traditional medicine, antibiotics, antipyretics and analgesics is common in Covid-19 patients [47-58].

4. Hepatic congestion: high levels of positive end expiratory pressure during mechanical ventilation can lead to elevated right atrial pressures and hepatic congestion [47-58].

\subsection{Clinical effect of SARS-CoV-2 in Rheumatoid Arthritis (RA)}

A study recently published in Autoimmunity Reviews has suggested that the combined effects of impaired immunity and the use of corticosteroids and immunosuppressive drugs increases the risk of bacterial and viral infections in RA patients [73, 74]. According to the Global Rheumatology Alliance Global Registry, patients preferably age $>65$-year-old with acute flares of active rheumatic disease, living in a health care facility and other associated comorbidities are at increased risk of severe illness. The Italian Society for Rheumatology performed a retrospective study to monitor impact of COVID-19 in patients with autoimmune rheumatic diseases. Carlo Alberto Scirè et al. (2020) recruited 232 (36\% males) elderly patients (mean age 62.2 years), who used corticosteroids $(51.7 \%)$ suffering from multiple comorbidities. Rheumatoid Arthritis was the most common disease (34.1\%), followed by spondyloarthritis $(26.3 \%)$, connective tissue disease (21.1\%) and vasculitis (11.2\%). 69.4\% patients had an active disease [74]. Typical COVID-19 clinical presentation occurs, with systemic symptoms (fever and lack of energy) and respiratory symptoms. The overall outcome was severe with $69.8 \%$ required hospitalization, 55.7 $\%$ required respiratory oxygen support, $20.9 \%$ needed invasive ventilation or $7.55 \%$ needed mechanical ventilation and $19 \%$ patients died. Male sex was a poor prognostic factor. Immunomodulatory treatments were not significantly associated with an increased risk of ICU admissions and death [74].

Along with other common respiratory symptoms of COVID-19, musculoskeletal symptoms can develop. In a recent study, arthralgia was reported in 15\% and myalgia in almost 44\% in COVID-19 patients, although COVID-19 is not well-known to cause clinical arthritis. However, high dose glucocorticoids diminish the musculoskeletal symptoms of COVID-19. We hypothesized that the COVID-19 pandemic could erroneously cause an increase in RA cases. There is still no report which indicates that people develop 
autoimmune inflammatory arthritis, such as RA, after being infected by SARS-CoV-2 or any of the other six known human coronaviruses $[65,75,76$, 77]

The release of pro-inflammatory cytokines in the alveolar membranes during severe COVID-19 infection share connections with the primary cytokines (IL-1, IL-6, IL-17, TNF) targeted in the treatment of RA. Both diseases induce extensive tissue destruction and organ failure. COVID-19 is characterized by a robust host immune response, elicited by alveolar epithelial cell damage and $\mathrm{T}$ cell activation in the lungs that causes uncontrolled local production of proinflammatory effector cytokines leading to the attraction of a vast majority of neutrophils and macrophages to the lungs. Barrier damage, T-cell activation, effector cytokine production, and neutrophil influx (the critical features of synovitis) are commonalities between COVID19 and RA. Whether cytokine inhibition can block this process remains to be established, but a rationale exists for this approach. The use of cytokine inhibitors in COVID-19 is balanced by the fact that a pathological hyper-inflammatory state is driving the pathology and might require therapeutic cytokine inhibition [78].

We initially assumed that immunocompromised patients with autoimmune rheumatic diseases were likely to present with a more severe course of COVID-19 requiring oxygen support and admission to ICU. However, the total prevalence of inflammatory rheumatic disorders among ICU patients with SARS-CoV-2 pneumonia was low $(1.1 \%)$ as compared to the general population $(1 \%-2 \%)$. Moreover, most critically ill patients with rheumatic disease had multiple comorbidities like cardiovascular diseases, obesity and diabetes mellitus that can contribute to the development of ARDS. That is why any definite conclusions regarding the risk of severe COVID-19 in rheumatic patients cannot be made, since we do not know the total number of infected patients with autoimmune rheumatic diseases in Russia. As a general rule, patients with any chronic illness are at increased risk of contracting respiratory infections frequent outpatient clinic visits or hospitals where they can have exposure with infected individuals [73].
No specific treatment for COVID-19 warrants symptomatic care, so the control of disease activity is the mainstay of management of RA patients during COVID-19. Also, the management of comorbidities and extra-articular features plays a crucial role in the control of the disease. Recommendations regarding the use of immunomodulatory drugs during COVID-19 are as follows [79, 80, 81].

- NSAIDs: May be used in active arthritis but stopped in case of severe respiratory symptoms with diagnosed or suspected COVID-19.

- Steroids: Corticosteroids are the mainstay of initial treatment in acute RA flares. Lowest-possible dexamethasone use is recommended in patients with moderate to severe COVID-19. Sudden discontinuation should be avoided.

- csDMARDS: May be used, but Sulfasalazine, Methotrexate and Leflunomide should be stopped in patients with diagnosed or suspected COVID-19.

- bDMARDS, tsDMARDS: May be considered in moderate to severe RA, JAK inhibitors and non-IL-6 biologics should be discontinued in patients with diagnosed or suspected COVID-19. Switching from intravenous to subcutaneous form can be considered if available. Increase of dosing-interval or reduction of RTX can be considered.

- Pneumococcal, influenza vaccine and Vitamin D: These are also recommended for suspected patients.

\subsection{Clinical effect of SARS-CoV-2 on Nervous System}

Although the predominant clinical presentation is with respiratory disease, neurological manifestations are being increasingly recognized [82]. Olds \& Kabbani (2020) raised the question of nicotine associated neurological comorbidity in COVID19 patients that the viral target receptor ACE2 is expressed in the brain and functionally interacts with nAChRs [83]. They considered neural cells and astrocytes (especially 
in the hypothalamus and brainstem) more prone to infection in smokers because nicotine stimulation of the $\mathrm{nAChR}$ was found to increase ACE2 expression within them. ACE2 signaling pathway is believed to counteract oxidative stress and neuroinflammation, thus, disruption in ACE balance can lead to neurodegeneration of dopaminergic neurons or impairment in cholinergic pathways which might participate in the progression of Alzheimer's disease [83].

For a given virus, the ability to infect certain cells, tissues, or even species while not affecting others is referred to as viral tropism. This viral tropism, allowing a virus to replicate in and affect certain body tissues, would then lead to the symptomatic presentation of that virus. A major factor that dictates this tissue selectivity is the virus' ability to bind and take over specific host cell surface receptors. Recent research on SARS-CoV-2 has shown that similarly to SARS-CoV, this virus can invade tissues by binding to the ACE2 receptor on certain host cells. It has been reported that the brain also expresses ACE2 receptors on glial cells and neurons and is most prominent in the brainstem, the paraventricular nucleus (PVN), nucleus tractus solitarius (NTS), and the rostral ventrolateral medulla which all play a role in cardiovascular regulation. [47, 84].

The neurological manifestations can be generally divided into two categories: central and peripheral. Central manifestations include headache, dizziness, impaired consciousness, encephalopathy, delirium, global confusion, syncope, seizures, gait difficulties, cerebrovascular events, encephalitis, and postinfectious autoimmunity. Peripheral disorders include isolated cranial nerve dysfunctions (i.e., impaired sense of smell and taste sensation), Guillain-Barré-syndrome, and myositis-like muscle injury. Although the majority of neurological symptoms develop throughout the course of illness, others such as acute strokes can be the initial presentation. [85] A systematic review of neuroimaging and neurologic manifestations of 214 patients in China suggested headache and dizziness were amongst the most common neurological signs of COVID-19. However, the role of MRI and CT scan of the brain has shown that once within the CNS,
COVID-19 is shown to produce more detrimental neurological manifestations; including but not limited to an aneurysm, encephalitis, polyneuropathy, anosmia, ageusia, ischemic and hemorrhagic strokes. COVID-19 isolation and contact precaution measures have limited ability to perform neurological complications early on. Therefore, it is recommended that patients with COVID-19 neurological conditions should be followed for some months after recovery to report any worsening of symptoms. [86]

As per Berger et al (2020), headache is a common symptom of COVID-19 being observed in up to $40 \%$ of patients [87]. Similarly, the contribution of CNS viral invasion by SARS-CoV-2 contributes to the frequently observed altered levels of consciousness in the severely ill COVID-19 patient as opposed to general systemic disease. Unsurprisingly, encephalopathy has been described. Patients with encephalopathy consequent to overwhelming infection and associated co-morbidities are likely to be the most common neurological complication. Several pathophysiological processes may be responsible for an increased risk of stroke with COVID-19. Severe disease is seen more often in older patients who often have comorbidities that increase their risk of stroke. [86] As per Zhou et al. (2020), markers of coagulation may be increased during infection, and disseminated intravascular coagulation has been observed [88]. Brain endothelial cells express ACE-2 receptors, and the potential exists for viral-induced vasculitis. Risk may be increased for both arterial and venous cerebrovascular disease. [46, 89, 90]

According to Seiden et al. (2004), loss of smell in association with upper respiratory infection is common and has been attributed damage to olfactory epithelial by the causative virus [91]. Anosmia has been reported in up to 30 to $66 \%$ of patients and may be an early symptom of infection. Anecdotally, in mild infection, it may be the only clue of infection. Anosmia is often accompanied by ageusia, or loss of taste [92]. Anosmia and ageusia related to COVID-19 typically resolve over several weeks. A high expression of ACE-2 receptor on oral mucosa 
has been reported and may be related to these symptoms with SARS-CoV-2 [47, 89].

\section{Conclusion}

The COVID-19 pandemic continues to threaten people globally and therefore the global economy due to its potential threat on the healthcare system. Patients with comorbidities are especially more prone to severe complications of COVID19 and higher rates of hospitalization. Comorbidities may range from any pre-existing cardiac, respiratory, gastrointestinal, endocrine, renal, autoimmune, or other immunocompromised state of a disease. During our literature review we learned about the pathophysiology of SARS-CoV-2 and how it affects multiple organ systems. Symptomatic and supportive care are all clinicians can provide at this time.

\section{Competing Interests}

The authors indicate no potential conflict of interest.

\section{How to Cite this Article:}

Bhatt et al., "COVID-19 Pathophysiology and Clinical Effects on Multiple Organ Systems - A Narrative Review”, Int. Ann. Sci., vol. 10, no. 1, pp. 151-167, Jun. 2021. https://doi.org/10.21467/ias.10.1.151-167

\section{References}

[1] China CDC Weekly (2020). Vital surveillance: The epidemiological characteristics of an outbreask of 2019. Novel Coronoavirus Disease (COVID-19). 2(8): 113122. doi: $10.46234 / \mathrm{ccdcw} 2020.032$ Accessed on April 25, 2021 https://scholar.harvard.edu/files/kleelerner/files/202002 17_ccdc_-

_the_epidemiological_characteristics_of_an_outbreak_ of_2019_novel_coronavirus_diseases_covid-19__china_cdc.pdf

[2] Zu, Z. Y., Jiang, M. D., Xu, P. P., Chen, W., Ni, Q. Q., Lu, G. M., \& Zhang, L. J. (2020). Coronavirus Disease 2019 (COVID-19): A Perspective from China. Radiology, 296(2), E15-E25. https://doi.org/10.1148/radiol.2020200490

[3] Rothan, H. A., \& Byrareddy, S. N. (2020). The epidemiology and pathogenesis of coronavirus disease (COVID-19) outbreak. Journal of autoimmunity, 109, 102433. https://doi.org/10.1016/j.jaut.2020.102433

[4] Renu K, Prasanna PL, Valsala Gopalakrishnan A. Coronaviruses pathogenesis, comorbidities and multiorgan damage - A review. Life Sci. 2020;255:117839. doi:10.1016/j.lfs.2020.117839

[5] Centers for Disease Control and Prevention. (2020). COVID-19 Associated Hospitalization Related to
Underlying Medical Conditions. Centers for Disease Control and Prevention. Accessed April 25, 2021 from https://www.cdc.gov/coronavirus/2019-ncov/coviddata/investigations-discovery/hospitalizationunderlying-medical-conditions.html

[6] Centers for Disease Control and Prevention. (2020, September 23). COVID-19 Provisional Counts - Weekly Updates by Select Demographic and Geographic Characteristics. Centers for Disease Control and Prevention. Accessed on April 25, 2020 from https://www.cdc.gov/nchs/nvss/vsrr/covid_weekly/inde x.htm?fbclid=IwAR3-wrg3tTKK5-

9tOHPGAHWFVO3DfslkJ0KsDEPQpWmPbKtp6Eso VV2Qs1Q.

[7] World Health Organization. (2020). Advice for the public on COVID-19. World Health Organization. Accessed on April 25, $2021 \quad$ from https://www.who.int/emergencies/diseases/novelcoronavirus-2019/advice-for-public

[8] Berardi, A., Perinelli, D. R., Merchant, H. A., Bisharat, L., Basheti, I. A., Bonacucina, G., ... Palmieri, G. F. (2020). Hand sanitisers amid CoViD-19: A critical review of alcohol-based products on the market and formulation approaches to respond to increasing demand. International Journal of Pharmaceutics, 584, 119431. https://doi.org/10.1016/j.ijpharm.2020.119431

[9] Eikenberry, S. E., Mancuso, M., Iboi, E., Phan, T., Eikenberry, K., Kuang, Y., ... Gumel, A. B. (2020). To mask or not to mask: Modeling the potential for face mask use by the general public to curtail the COVID-19 pandemic. Infectious Disease Modelling, 5, 293-308. https://doi.org/10.1016/j.idm.2020.04.001

[10] Gandhi,M., Beyrer,C., Goosby,E. (2020). Masks do more than protect others during COVID-19: reducing the inoculum of SARS-CoV-2 to protect the wearer. Journal of General Internal Medicine. 2020 Oct, 35(10): 30633066

[11] Zhi, Z. (2020). The epidemiological characteristics of an outbreak of 2019 novel coronavirus diseases (COVID19) in China. Retrieved September 14, 2020, from https://pubmed.ncbi.nlm.nih.gov/32064853/

[12] WHO. (2020). Novel Coronavirus (2019-nCoV) Situation Report-1. Retrieved September 14, 2020, from https:// www.who.int/docs/defaultsource/coronaviruse/situationreports/20200121-sitrep-12019-ncov.pdf?sfvrsn=20a99c10_4

[13] WHO. (2020). Coronavirus disease 2019 (COVID-19) Situation Report-26. Retrieved September 14, 2020, from https:// www.who.int/docs/defaultsource/coronaviruse/situationreports/20200215-sitrep26-covid-19.pdf?sfvrsn=a4cc6787_2

[14] Holshue, M., DeBolt, C., Lindquist, S., Lofy, K., Wiesman, J., Bruce, H. et al. First case of 2019 novel coronavirus in the united states. New England Journal of Medicine 2020; 382: 929-936. Doi: 10.1056/NEJMoa2001191

[15] Bashir MF, Ma B, Bilal, Komal B, Bashir MA, Tan D, Bashir M. Correlation between climate indicators and COVID-19 pandemic in New York, USA. Sci Total Environ. 2020 Aug 1;728:138835. doi: 10.1016/j.scitotenv.2020.138835. Epub 2020 Apr 20. PMID: 32334162; PMCID: PMC7195034.

[16] Zimmermann P, Curtis N. Coronavirus Infections in Children Including COVID-19: An Overview of the Epidemiology, Clinical Features, Diagnosis, Treatment and Prevention Options in Children. Pediatr Infect Dis J. 2020 May;39(5):355-368. doi: 
Bhatt et al., Int. Ann. Sci.; Vol. 10, Issue 1, pp: 151-167, 2021

10.1097/INF.0000000000002660. PMID: 32310621; PMCID: PMC7158880.

[17] WHO (2020). Coronavirus disease (COVID-19) weekly epidemiological update. Retrieved September 15, 2020, from https://www.who.int/docs/defaultsource/coronaviruse/situation-reports/20200914weekly-epi-update-5.pdf?sfvrsn=cf929d04_2

[18] Zhai P, Ding Y, Wu X, Long J, Zhong Y, Li Y. The epidemiology, diagnosis and treatment of COVID-19. Int J Antimicrob Agents. 2020 May;55(5):105955. doi: 10.1016/j.ijantimicag.2020.105955. Epub 2020 Mar 28. PMID: 32234468; PMCID: PMC7138178.

[19] Yang, Y., Lu, Q., Liu, M., Wang, Y., Zhang, A., Jalali, N., et al. (2020) Epidemiological and clinical features of the 2019 novel coronavirus outbreak in China. MedRxiv preprint

doi: https://doi.org/10.1101/2020.02.10.20021675

[20] Zhang, H., Penninger, J., Li, Y., Zhong N., Slutsky, A. Angiotensin-converting enzyme 2 (ACE2) as a SARSCoV-2 receptor: molecular mechanisms and potential therapeutic target. Intensive Care Med (2020) 46: 586590

[21] Hu, Y., Sun, J., Dai, Z., et al. Prevalence and severity of corona virus disease 2019 (COVID-19): A systematic review and meta-analysis. J Clin Viro. 2020 Jun; 127:104371. Doi:10.1016j.jcv.2020.10447

[22] Chu, H., Chan, JF., Wang, Y., Yen TT., et al. Comparative replication and Immune Activation Profile of SARS-CoV-2 and SARS-CoV in Human Lungs: An Ex Vivo Study with implications for the pathogenesis of COVID-19. Clin Infect Dis. 2020 Sep 12;71(6): 1400-1409.

Doi:10.1093/cid/ciaa410.PMID:32270184;

PMCID:PMC7184390.

[23] Ackermann, M., Verleden, S., Kuehnel, M., Haverich, A. Pulmonary Vascular Endothelialitis, Thrombosis, and Angiogenesis in COVID-19. N Engl J Med 2020; 383:120-128. DOI 10.1056NEJMoa2015432

[24] Guoping, L., Xiang, H., Zhang, L, Ran, Q. et al. Assessing ACE2 expression patterns in lung tissues in the pathogensis of COVID-19. J Autoimmun. 2020 Aug; 112:102463. Doi 10.1016/j.jaut.2020.102463.

[25] Li, X., Ma, X. Acute respiratory failure in COVID-19: is it "typical" ARDS? Crit Care. 2020; 24: 198. Doi 10.1186/s13054-020-02911-9.

[26] American Lung Association (2020). What is COVID-19. Retrieved on April 25, 2021 from https://www.lung.orlung-health-disease/lung-diseaselookup/covid-19/about-covid-19 Accessed September 30, 2020

[27] Zheng YY, Ma YT, Zhang JY, Xie X. COVID-19 and the cardiovascular system. Nat Rev Cardiol. 2020 May;17(5):259-260. doi: 10.1038/s41569-020-0360-5. PMID: 32139904; PMCID: PMC7095524.

[28] Bansal M. Cardiovascular disease and COVID-19. Diabetes Metab Syndr. 2020 May-Jun;14(3):247-250. doi: 10.1016/j.dsx.2020.03.013. Epub 2020 Mar 25. PMID: 32247212; PMCID: PMC7102662.

[29] Kang Y, Chen T, Mui D, Ferrari V, Jagasia D, ScherrerCrosbie M, Chen Y, Han Y. Cardiovascular manifestations and treatment considerations in COVID19. Heart. 2020 Aug;106(15):1132-1141. doi: 10.1136/heartjnl-2020-317056. Epub 2020 Apr 30. PMID: 32354800; PMCID: PMC7211105.

[30] Li B, Yang J, Zhao F, Zhi L, Wang X, Liu L, Bi Z, Zhao Y. Prevalence and impact of cardiovascular metabolic diseases on COVID-19 in China. Clin Res Cardiol. 2020
May;109(5):531-538. doi: 10.1007/s00392-020-016269. Epub 2020 Mar 11. PMID: 32161990; PMCID: PMC7087935.

[31] Ye, Q., Wang, B., Mao, J. The pathogenesis and treatment of the 'Cytokine Storm' in COVID-19. J Infect. 2020 Jun; 80(6): 607-613.

[32] Sinha, P., Matthay, M., Calfee, C. Is a "Cytokine Storm" relevant to COVID-19? JAMA Intern Med. 2020;180(9):1152-1154.

doi:10.1001/jamainternmed.2020.3313

[33] Bhaskar, S., Sinha, A., Banach, M., Mittoo, S., Weissert, R., Kass, J., Rajagopal, S., Pai, A., Kutty, S. Cytokine Storm in COVID-19-Immunopathological Mechanisms, Clinical Considerations, and Therapeutic Approaches: The REPROGRAM Consortium Position Paper. Front Immunol. 2020; 11: 1648.

[34] Mangalmurti, M. \& Hunter, C. Cytokine Storms: Understanding COVID-19. Immunity. $2020 \mathrm{Jul}$ 14; 53(1): 19-25.

[35] Sun, X., Wang, T., Cai, D., Hu, Z., Chen, J. et al. Cytokine storm intervention in the early stages of COVID-19 pneumonia. Cytokine Growth Factor Rev. 2020 Jun; 53: 38-42.

[36] Matthay MA, Aldrich JM, Gotts JE. Treatment for severe acute respiratory distress syndrome from COVID-19. Lancet Respir Med 8: 433-434, 2020. doi:10.1016/S2213-2600(20)30127-2.

[37] Wu C, Chen X, Cai Y, Xia J, Zhou X, Xu S, Huang H, Zhang L, Zhou X, Du C, Zhang Y, Song J, Wang S, Chao Y, Yang Z, Xu J, Zhou X, Chen D, Xiong W, Xu L, Zhou F, Jiang J, Bai C, Zheng J, Song Y. Risk Factors Associated With Acute Respiratory Distress Syndrome and Death in Patients With Coronavirus Disease 2019 Pneumonia in Wuhan, China. AMA Intern Med. 2020;180(7):934-943.

doi:10.1001/jamainternmed.2020.0994. PMID: 32167524, PMCID: PMC7070509

[38] Batlle D, Soler MJ, Sparks MA, Hiremath S, South AM, Welling PA, Swaminathan S .COVID-19 and ACE2 in Cardiovascular, Lung, and Kidney Working Group. Acute Kidney Injury in COVID-19: Emerging Evidence of a Distinct Pathophysiology.Journal of the American Society of Nephrology, July 2020, 31(7): 1380-1383

[39] Husain-Syed et al, F., Slutsky, A. S. \& Ronco, C. Lungkidney cross-talk in the critically ill patient. Am. J. Respir. Crit. Care Med. 194, 402-414 (2016)

[40] Ronco C , Reis T. Kidney involvement in COVID-19 and rationale for extracorporeal therapies. Nat Rev Nephrol 2020; $16: 308-310$

[41] Hirsch JS , Ng JH , Ross DW et al. . Acute kidney injury in patients hospitalized with COVID-19. Kidney Int 2020; 98: 209-218

[42] Gabarre P, Dumas G, Dupont T, Darmon M, Azoulay E, and Zafrani L. Acute kidney injury in critically ill patients with COVID-19. Intensive Care Med 46, 13391348 (2020). doi: 10.1007/s00134-020-06153-9. Epub 2020 Jun 12. PMID: 32533197; PMCID: PMC7290076.

[43] Ronco C, Reis T, Husain-Syed F. Management of acute kidney injury in patients with COVID-19. Lancet Respir Med. 2020 Jul; 8(7): 738-742. PMID: 32416769; PMCID: PMC7255232.

[44] Wang D, Hu B, Hu C, Zhu F, Liu X, Zhang J, Wang B. et al. Clinical characteristics of 138 hospitalized patients with 2019 novel coronavirus-infected pneumonia in Wuhan, China. JAMA. 2020;323(11):1061-1069. doi: 10.1001/jama.2020.1585. 
[45] Tadic, M., Cuspidi, C., \& Sala, C. (2020). COVID-19 and diabetes: Is there enough evidence? Journal of clinical hypertension (Greenwich, Conn.), 22(6), 943948. https://doi.org/10.1111/jch.13912

[46] Lian, J., Jin, X., Hao, S., Cai, H., Zhang, S., Zheng, L., Jia, H., Hu, J., Gao, J., Zhang, Y., Zhang, X., Yu, G., Wang, X., Gu, J., Ye, C., Jin, C., Lu, Y., Yu, X., Yu, X., Ren, Y., ... Yang, Y. (2020). Analysis of Epidemiological and Clinical Features in Older Patients With Coronavirus Disease 2019 (COVID-19) Outside Wuhan. Clinical infectious diseases : an official publication of the Infectious Diseases Society of America, 74(15), 740-747. https://doi.org/10.1093/cid/ciaa242

[47] Hoffmann, M., Kleine-Weber, H., Schroeder, S., Krüger, N., Herrler, T., Erichsen, S., Schiergens, T. S., Herrler, G., Wu, N. H., Nitsche, A., Müller, M. A., Drosten, C., \& Pöhlmann, S. (2020). SARS-CoV-2 Cell Entry Depends on ACE2 and TMPRSS2 and Is Blocked by a Clinically Proven Protease Inhibitor. Cell, 181(2), 271-280.e8. https://doi.org/10.1016/j.cell.2020.02.052

[48] Fang, L., Karakiulakis, G., \& Roth, M. (2020). Are patients with hypertension and diabetes mellitus at increased risk for COVID-19 infection?. The Lancet. Respiratory medicine, 8(4), e21. https://doi.org/10.1016/S2213-2600(20)30116-8

[49] Guo, W., Li, M., Dong, Y., Zhou, H., Zhang, Z., Tian, C., Qin, R., Wang, H., Shen, Y., Du, K., Zhao, L., Fan, H., Luo, S., \& Hu, D. (2020). Diabetes is a risk factor for the progression and prognosis of COVID-19. Diabetes/metabolism research and reviews, e3319. Advance online publication. https://doi.org/10.1002/dmrr.3319

[50] Patel, K. P., Patel, P. A., Vunnam, R. R., Hewlett, A. T., Jain, R., Jing, R., \& Vunnam, S. R. (2020). Gastrointestinal, hepatobiliary, and pancreatic manifestations of COVID-19. Journal of clinical virology : the official publication of the Pan American Society for Clinical Virology, 128, 104386. https://doi.org/10.1016/j.jcv.2020.104386

[51] Muniyappa \& Gubbi (2020, April 26). COVID-19 pandemic, coronaviruses, and diabetes mellitus: American Journal of Physiology, endocrinology and metabolism. Retrieved from: <https://doi.org/10.1152/ajpendo.00124.2020>

[52] Kumar, A., Arora, A., Sharma, P., Anikhindi, S. A., Bansal, N., Singla, V., Khare, S., \& Srivastava, A. (2020). Is diabetes mellitus associated with mortality and severity of COVID-19? A meta-analysis. Diabetes \& metabolic syndrome, 14(4), 535-545. https://doi.org/10.1016/j.dsx.2020.04.044

[53] Singh, A. K., Gupta, R., Ghosh, A., \& Misra, A. (2020). Diabetes in COVID-19: Prevalence, pathophysiology, prognosis and practical considerations. Diabetes \& metabolic syndrome, 14(4), 303-310. https://doi.org/10.1016/j.dsx.2020.04.004

[54] Bhaskar S, Rastogi A, Chattu VK, et al. Key Strategies for Clinical Management and Improvement of Healthcare Services for Cardiovascular Disease and Diabetes Patients in the Coronavirus (COVID-19) Settings: Recommendations From the REPROGRAM Consortium. Front Cardiovasc $\quad$ Med. 2020;7:112. Published 2020 Jun 16. doi:10.3389/fcvm.2020.00112

[55] Barclay L, Nyarko E. Are Diabetes, CVD Associated with Worse COVID-19 Prognosis? Medscape (2020). Available

online at: https://www.medscape.org/viewarticle/926097 (acce ssed April 5, 2020).

[56] Ahmed, W. N., Arun, C. S., Koshy, T. G., Nair, A., Sankar, P., Rasheed, S. A., \&amp; Ann, R. (2020). Management of diabetes during fasting and COVID-19 Challenges and solutions. Journal of family medicine and primary care, 9(8), 3797-3806.

[57] Gu, J., Han, B., \& Wang, J. (2020). COVID-19: Gastrointestinal Manifestations and Potential Fecal-Oral 854 Transmission. Gastroenterology, 158(6), 15181519. https://doi.org/10.1053/j.gastro.2020.02.054

[58] Cheong, J., Bartell, N., Peeraphatdit, T., Mosli, M., \& AlJudaibi, B. (2020). Gastrointestinal and liver 856 manifestations of COVID-19. Saudi journal of gastroenterology : official journal of the Saudi 857 Gastroenterology Association, 26(5), 226-232. https://doi.org/10.4103/sjg.SJG_147_20

[59] Cha, M. H., Regueiro, M., \& Sandhu, D. S. (2020). Gastrointestinal and hepatic manifestations of COVID19: 859 A comprehensive review. World journal of gastroenterology, 26(19), 2323-2332. 860 https://doi.org/10.3748/wjg.v26.i19.2323

[60] Baryah, A., Midha, V., Mahajan, R., \& Sood, A. (2020). Impact of Corona Virus Disease-19 (COVID-19) 862 pandemic on gastrointestinal disorders. Indian journal of gastroenterology : official journal of the Indian 863 Society of Gastroenterology, 39(3), 214-219. https://doi.org/10.1007/s12664-020-01071-6

[61] Kotfis, K., \& Skonieczna-Żydecka, K. (2020). COVID19: gastrointestinal symptoms and potential sources of 865 SARS-CoV-2 transmission. Anaesthesiology intensive therapy, 52(2), 171-172. 866 https://doi.org/10.5114/ait.2020.93867

[62] Téllez L, Martín Mateos RM. COVID-19 and liver disease: An update [Actualización en COVID-19 y enfermedad hepática]. Gastroenterología y Hepatología (English Edition). 2020;43(8):472-480. doi:10.1016/j.gastre.2020.06.005

[63] Cai Q, Huang D, Ou P, Yu H, Zhu Z, Xia Z, Su Y, Ma Z, Zhang Y, Li Z, He Q, Liu L, Fu Y, Chen J. COVID19 in a designated infectious diseases hospital outside Hubei Province, China. Allergy. 2020 Jul;75(7):17421752. doi: 10.1111/all.14309. Epub 2020 Apr 17. PMID: 32239761 .

[64] Fan Z, Chen L, Li J, Cheng X, Yang J, Tian C, Zhang Y, Huang S, Liu Z, Cheng J. Clinical Features of COVID19-Related Liver Functional Abnormality. Clin Gastroenterol Hepatol. 2020 Jun;18(7):1561-1566. doi: 10.1016/j.cgh.2020.04.002. Epub 2020 Apr 10. PMID: 32283325; PMCID: PMC7194865.

[65] Guan W-j, Ni Z-y, Hu Y, Liang W-h, Ou C-q, He J-x, et al. Clinical characteristics of coronavirus disease 2019 in China. New England journal of medicine. 2020;382(18):1708-20.

[66] Li L, Li S, Xu M, Yu P, Zheng S, Duan Z, et al. Risk factors related to hepatic injury in patients with corona virus disease 2019. March 2020. medRxiv preprint doi: https://doi.org/10.1101/2020.02.28.20028514;

[67] Liu, Wei1; Tao, Zhao-Wu2; Wang, Lei1; Yuan, MingLi1; Liu, Kui3; Zhou, Ling3; Wei, Shuang3; Deng, Yan3; Liu, Jing4; Liu, Hui-Guo3; Yang, Ming5; Hu, Yi1 Analysis of factors associated with disease outcomes in hospitalized patients with 2019 novel coronavirus disease, Chinese Medical Journal: May 5, 2020. 133(9): 1032-1038 doi: 10.1097/CM9.0000000000000775

[68] Shi H, Han X, Jiang N, Cao Y, Alwalid O, Gu J, et al. Radiological findings from 81 patients with COVID-19 
pneumonia in Wuhan, China: a descriptive study. The Lancet Infectious Disease. April 01, 2020. 20(4): 425434. DOI:https://doi.org/10.1016/S14733099(20)30086-4

[69] Wu J, Li W, Shi X, Chen Z, Jiang B, Liu J, Wang D, Liu C, Meng Y, Cui L, Yu J, Cao H, Li L. Early antiviral treatment contributes to alleviate the severity and improve the prognosis of patients with novel coronavirus disease (COVID-19). J Intern Med. 2020 Jul;288(1):128138. doi: 10.1111/joim.13063. Epub 2020 Apr 20. PMID: 32220033.

[70] Zhang Y, Zheng L, Liu L, Zhao M, Xiao J, Zhao Q. Liver impairment in COVID-19 patients: A retrospective analysis of 115 cases from a single centre in Wuhan city, China. Liver Int. 2020 Sep;40(9):2095-2103. doi: 10.1111/liv.14455. Epub 2020 Apr 28. PMID: 32239796.

[71] Xu Z, Shi L, Wang Y, Zhang J, Huang L, Zhang C, et al. Pathological findings of COVID-19 associated with acute respiratory distress syndrome. The Lancet respiratory medicine. 2020;8(4):420-2.

[72] Chau TN, Lee KC, Yao H, Tsang TY, Chow TC, Yeung YC, et al. SARS-associated viral hepatitis caused by a novel coronavirus: report of three cases. Hepatology. 2004;39(2):302-10

[73] Moiseev S, Avdeev S, Brovko M, et al Rheumatic diseases in intensive care unit patients with COVID-19 (2020). Annals of the Rheumatic Diseases. Published as doi: 10.1136/annrheumdis-2020-217676

[74] Scirè CA, Carrara G, Zanetti A, Landolfi G, Chighizola C, Alunno A, Andreoli L, Caporali R, Gerli R, Sebastiani GD, Valesini G, Sinigaglia L; Italian Registry of the Italian Society for Rheumatology (CONTROL-19). COVID-19 in rheumatic diseases in Italy: first results from the Italian registry of the Italian Society for Rheumatology (CONTROL-19). Clin Exp Rheumatol. 2020 Jul-Aug;38(4):748-753. Epub 2020 Jul 28. PMID: 32723435 .

[75] Friedman, N. et al. Human coronavirus infections in Israel: epidemiology, clinical symptoms and summer seasonality of HCoV-HKU1. Viruses 10, 515 (2018).

[76] Christian, M. D., Poutanen, S. M., Loutfy, M. R., Muller, M. P. \& Low, D. E. Severe acute respiratory syndrome. Clin. Infect. Dis. 38, 1420-1427 (2004).

[77] Li, S. et al. Symptom combinations associated with outcome and therapeutic effects in a cohort of cases with SARS. Am. J. Chin. Med. 34, 937-947 (2006).

[78] Schett, G., Manger, B., Simon, D. et al. COVID-19 revisiting inflammatory pathways of arthritis. Nat Rev Rheumatol 16, 465-470 (2020). https://doi.org/10.1038/s41584-020-0451-z

[79] Mikuls R, Johnson SR, Fraenkel L, Arasaratnam RJ, Baden LR, Bermas BL, et al. (2020) American College of Rheumatology Guidance for the management of adult patients with rheumatic disease during the COVID-19 pandemic. Arthritis Rheumatology. September 2020, 72(9): e1-e12. https://doi.org/10.1002/art.41437

[80] Favalli E.G., Biggioggero M., Meroni P.L. Methotrexate for the treatment of rheumatoid arthritis in the biologic era: still an "anchor" drug? Autoimmun Rev. 2014;13:1102-1108. doi: 10.1016/j.autrev.2014.08.026

[81] Favalli E.G., Raimondo M.G., Becciolini A., Crotti C., Biggioggero M., Caporali R. The management of firstline biologic therapy failures in rheumatoid arthritis: current practice and future perspectives. Autoimmun Rev. 2017; 16: 1185-1195.

Doi: 10.1016/j.autrev.2017.10.002.
[82] Mark A Ellulet al (2020) Neurological associations of Covid-19. Lancet Neurol 2020; 19: 767-83 https://doi.org/10.1016/ S1474-4422(20)30221-0

[83] Olds JL, Kabbani N. Is nicotine exposure linked to cardiopulmonary vulnerability to COVID-19 in the general population? FEBS J. 2020 Sep;287(17):36513655. Doi: 10.1111/febs.15303. Epub 2020 Mar 28. PMID: 32189428; PMCID: PMC7228237.

[84] McFadden G. Cytokine determinants of viral tropism. Nat. Rev. Immunol. 2009;9(9):645-655.

[85] Zhou L, Zhang M, Wang J, Gao J. Sars-Cov-2:

Underestimated damage to nervous system. Travel Med Infect Dis. 2020; 36: 101642.

Doi:10.1016/j.tmaid.2020.101642

[86] Mao, L., Jin, H., Wang, M., Hu, Y., Chen, S., He, Q., et al. Neurologic manifestations of hospitalized patients with coronavirus disease 2019 in Wuhan, China. JAMA Neurology, 2021; 77(6): 683-690

[87] Berger J. R. (2020). COVID-19 and the nervous system. Journal of neurovirology, 26(2), 143-148. https://doi.org/10.1007/s13365-020-00840-5

[88] Zhou F, Yu T, Du R, Fan G, Liu Y, Liu Z, et al. (2020) Clinical course and risk factors for mortality of adult inpatients with COVID-19 in Wuhan, China: a retrospective cohort study. Lancet. March 28, 2020. 395(10229): 1054-1062. https://doi.org/10.1016/S01406736(20)30566-3

[89] Pereira A. Long-Term Neurological Threats of COVID19: A Call to Update the Thinking About the Outcomes of the Coronavirus Pandemic. Front Neurol. 2020;11:308. Published 2020 Apr 17. Doi:10.3389/fneur.2020.00308

[90] Xu H, Zhong L, Deng J, Peng J, Dan H, Zeng X et al (2020) High expression of ACE2 receptor of 2019-nCoV on the epithelial cells of oral mucosa. Int J Oral Sci. 12(1):8

[91] Seiden AM. Postviral olfactory loss. Otolaryngol Clin N Am. 2004;37(6):1159-1166. doi: $10.1016 /$ j.otc.2004.06.007.

[92] Hopkins C, Kumar N (2020) Loss of sense of smell as a marker of COVID-19 infection. ENTUK [cited 2020 March 30]; Accessed April 25, 2021 from https://www.entuk.org/sites/default/files/files/Loss $\% 20$ of $\% 20$ sense $\% 20$ of $\% 20$ smell $\% 20$ as $\% 20$ marker\%20of\% 20COVID.pdf

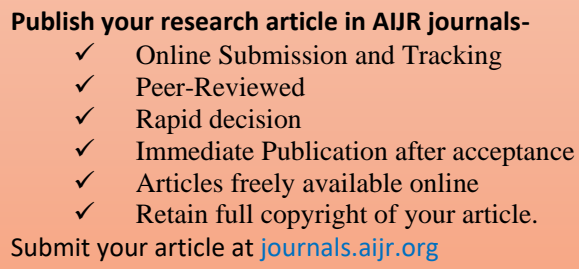

Publish your books with AIJR publisher-

$\checkmark \quad$ Publish with ISBN and DOI.

$\checkmark$ Publish Thesis/Dissertation as Monograph.

$\checkmark$ Publish Book Monograph.

$\checkmark \quad$ Publish Edited Volume/ Book.

$\checkmark \quad$ Publish Conference Proceedings

$\checkmark \quad$ Retain full copyright of your books.

Submit your manuscript at books.aijr.org 